\section{Constraining noble gas solubilities in the Earth's mantle}

\author{
ANGELIKA DOROTHEA ROSA ${ }^{1}$, TETSUO IRIFUNE ${ }^{2}$, \\ GUILLAUME MORARD ${ }^{3}$, AGNES DEWAELE ${ }^{4}$, GASTON \\ GARBARINO $^{5}$ AND ALI BOUHIFD $^{6}$
}

${ }^{1}$ European Synchrotron Radiation Facility

${ }^{2}$ Ehime University, Japan

${ }^{3}$ University Joseph Fourier

${ }^{4} \mathrm{CEA}$

${ }^{5} \mathrm{ESRF}$

${ }^{6} \mathrm{CNRS}$

Presenting Author: angelika.rosa@esrf.fr

Noble gases (NGs) are important geochemical markers for determining the volatile origins and their redistribution processes since Earth accretion. In order to reconstruct such processes, NG solubility and partitioning data between mineral and melts are required at Earth's mantle conditions. However, such information is difficult to obtain from field studies due to the potential atmospheric NG contamination, the few available deep Earth samples and the potential re-distribution of NGs during obduction.

In this contribution, we present an original approach to predict and constrain solubility behaviors of NGs at Earth's mantle conditions. Our previous experimental studies of the thermoelastic properties of heavy NGs ( $\mathrm{Ar}, \mathrm{Kr}, \mathrm{Xe})$ revealed extremely high compressibilities and high thermal expansivities of these elements [1-5]. We indeed observed a reduction of the atomic volume of NGs by $40 \%$ at transition zone depth. We propose that this may considerably affect the NG partitioning behaviors with increasing depth. In order to assess the influence of mechanical property changes on NG solubilities, we have extended the classical lattice strain approach, which is widely used to predict trace element partitioning between mineral and melt, by taking into consideration the mechanical properties of NGs and the one of the host phase. Using this approach, we have obtained solubilities that are in good agreement with those determined experimentally in lower mantle mineral phases [6]. In this contribution, we discuss the advantages and limitations of this approach and its application to upper mantle minerals, hydrous minerals and melts.

[1] Rosa et al., 2018, E equation of state of solid krypton up to 140 GPa, PRB, 97,035106.

[2] Rosa et al., submitted PRB, Compressional anomalies in solid krypton and xenon and equation of states.

[3] Dewaele et al., in prep., Equation of state and high pressure fcc to hcp transition of solid argon unraveled.

[4] Rosa et al., in prep., Thermal equation of state of xenon.

[5] Dewaele, et al., 2008, High pressure-high temperature equations of state of neon and diamond, Physical Review, 77(9).

[6] Rosa et al., 2020, Krypton storage capacity of the Earth's lower mantle, EPSL, 532, 116032.

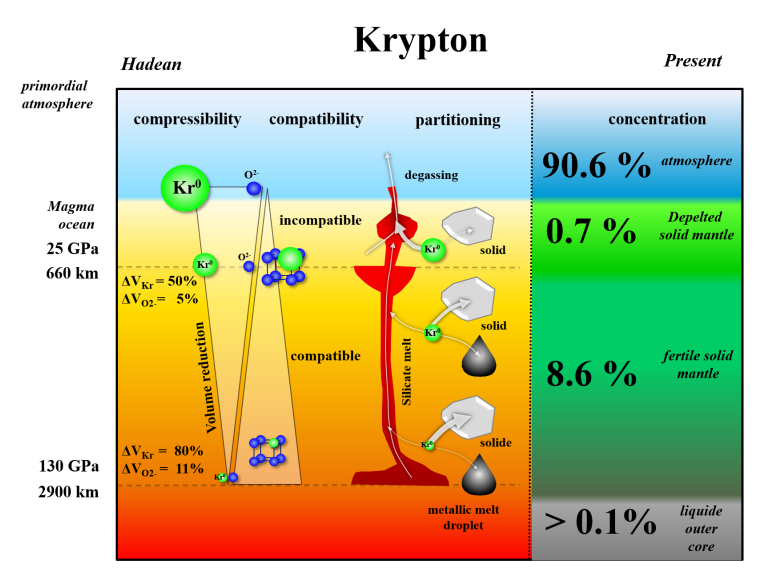

